over, the sliding spark is stable in space and more steady than the Millikan sparks; it works without any adjustments at all during many hours (for a frequency of two sparks a second).

The spectra we have obtained with copper electrodes (separation $3 \mathrm{~mm}$.), between 100 and $2000 \mathrm{~A}$., are, at first sight, similar to those given by ordinary vacuum sparks, except that some carbon lines are also present. From our results, it appears that 'sliding sparks' in vacuum will probably be useful as light sources for far ultra-violet spectrography. The spectrographic study of various types of 'sliding sparks' in vacuum is now in progress.

B. VODAR

N. AstoIn

Faculté des Sciences de Paris,

Laboratoire de Physique,

1 Rue Victor-Cousin,

Paris, 5e.

July 27.

${ }^{1}$ Millikan and Sawyer, Phys. Rev., 12, 167 (1918); Science, 50, 138 (1919). Bombke, "Vakuum spektrocospie"' (Leipzig, 1937). Vodar, Progres recents dans letude du spectre 0 . . Centre de perfectic

\section{Muscle Fibres of the Rat Diaphragm}

IN the course of an investigation of nerve endings in the rat diaphragm, it was noticed by Dr. J. A. Hewitt and one of us (C. S.) that the muscle fibres seemed to be of two types, reminiscent somewhat of the well-known pale and red fibres in voluntary muscles described by Jordan 1 and several others. The 'red' variety seemed to be more numerous than the other. Staining with iron hæmatoxylin after alcohol fixation (ideal for skeletal muscle) confirmed that there were two types, but unfortunately did not reveal any further distinguishing detail. It was then decided to attempt their differentiation at the higher magnifications now possible with the electron microscope.

The technique of specimen preparation was similar to that described by Hall, Jakus and Schmitt ${ }^{2}$, but was slightly modified to suit our particular tissue. A three-stage electron microscope built under the direction of Prof. G. I. Finch was used for this work, with an operating voltage of $80-120 \mathrm{kV}$.

For the first part of our work specimens were prepared from fresh pieces of diaphragm in the relaxed state. Krause's membrane is not confined to one myofibril, but is continued through those adjacent to it. The myosin filaments can be resolved in some specimens but not all, and extend throughout the length of the fibril. No limiting membrane can be seen surrounding the myofibril.

Two types of fibril were again observed in the electron microscope, which showed that though they are similar in most respects, they differ in their sarcomere lengths, especially after passive stretching

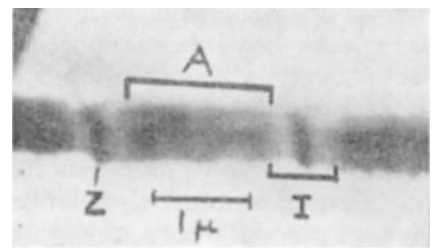

Fig. 1. Electron micrograph of a myofibril from rat diaphragm in the relaxed state (extending). This is due to a difference in the relative extensibilities of the $A$ and $I$ bends of the two fibrils, which is presumably a result of the difference in the structure of their $A$ bands, since there is independent evidence that the $I$ bands are similar, whereas the $A$ bands are different in their staining reaction.

For the second part of our work we compared stimulated diaphragm muscle fibrils in both the contracted and the relaxed states. The former was obtained by stimulating the phrenic nerve, allowing the fibres to contract isotonically and fixing them in this condition. The latter was obtained by stimulating, as before, another piece of diaphragm, and after isotonic contraction the stimulus was stopped; the muscle then lengthened to the relaxed state in which it was fixed.

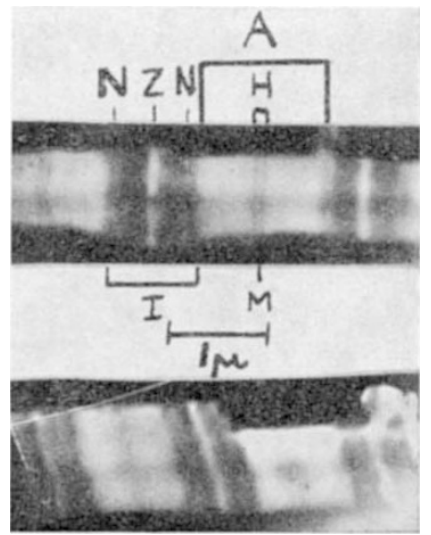

(a)

Fig. 2. Electron micrographs of flbrils from (a) rat rectus and (b) rat diaphragm, both after stimulation. The $\boldsymbol{H}$ disk and the

The fibrils from stimulated diaphragm exhibit more detail than those from one not so stimulated. The $A$ band now shows Hensen's disk with the $M$ line within it, and the $I$ band shows the $N$ lines in some fibrils.

Changes in sarcomere lengths in both 'pale' and 'red' fibres occurring of necessity in isotonic contraction are seen most noticeably in the lengths of the $I$ bands.

Our observations are in agreement with those reported by Hall et al. ${ }^{2}$ on leg muscles of the rabbit.

A few observations were made on the rectus muscle of the rat, which indicate that similar structural alterations take place after stimulation.

A. fuller account of this work will be published elsewhere. We wish to thank Prof. G. I. Finch for his help and for giving us facilities in his laboratory, and Dr. J. A. Hewitt for suggesting the original work and for criticism.

\section{SitTaRamayya}

Department of Physiology,

King's College,

London, W.C.2.

\section{M. Вцбнм}

Applied Physical Chemistry Laboratory, Imperial College of Science and Technology, London, S.W.7. Sept. 15.

1 Jordan, H. E., Physiol. Rev., 18, 301 (1933).

${ }^{2}$ Hall, C. E., Jakus, M. A., and Schmitt, F. O., Biol. Bull., 80, 32 